\title{
New innovations in craniomaxillofacial fixation: the 2.0 lock system
}

\author{
Brian Alpert, Rolf Gutwald ${ }^{1}$ and Rainer Schmelzeisen ${ }^{1}$ \\ Departments of Oral \& Maxillofacial Surgery and Surgical \& Hospital Dentistry, University of Louisville School \\ of Dentistry, Louisville, KY, USA, ${ }^{1}$ Department of Oral \& Maxillofacial Surgery, Albert-Ludwigs-University \\ Freiburg, Freiburg, Germany
}

(Received for publication on October 7, 2002)

\begin{abstract}
Rigid internal fixation with plates and screws is now standard for the treatment of fractures, osteotomies and reconstruction of the craniomaxillofacial skeleton. The latest innovations are selfdrilling, self-tapping screws and locking miniplates. These screws offer the prospect of less instrumentation and faster application. Preclinical testing has shown them to be substantially more retentive in cancellous bone, a significant advance in cancellous block bone grafting. Locking 2.0 miniplates utilize double threaded screws which both lock to the bone and the plate creating a mini-internal fixator. This results in a more rigid construct with less distortion of the fracture or osteotomy, screws which do not loosen and less interference with bone circulation since the plate is not pressed tightly against the bone. Locking miniplates are designed for midface application in the repair of fractures, osteotomies and defects. Three configurations in a variety of shapes and lengths are available for mandibular surgery. The thinner and medium varieties are useful in transoral plating of fractures utilizing the Champy technique. The heavier, longer variety are used in unilateral edentulous fractures in the symphysis and parasymphysis as well as an aid to tumor resection and reconstruction with both free and vascularized grafts. They are not designed to replace the heavier 2.4 locking reconstruction plates designed for complex fractures or extensive reconstructions. (Keio J Med 52 (2): 120-127, June 2003)
\end{abstract}

Key words: locking miniplates, self-drilling screws

Over the past 20 years, rigid internal fixation with plates and screws has become the standard for the treatment of fractures, osteotomies and reconstruction of the craniomaxillofacial skeleton. The devices continue to evolve with the latest innovations being selfdrilling, self-tapping screws and locking miniplates.

\section{Self-Drilling, Self-Tapping Screws}

These devices have been developed by AO/ASIF have undergone extensive in vitro as well as in vivo testing in both the laboratory and extensive clinical trials in both Europe and the United States. The first innovation involves self-drilling, self-tapping screws. Though not a new innovation, the AO version has been refined with a new self-retaining star drive deliv- ery system. Preclinical investigations at the AO center utilizing cadaver cortical bone in 1 to $3 \mathrm{~mm}$ thicknesses as well as cancellous bone block have demonstrated that in thin bone, (1 to $3 \mathrm{~mm}$ thickness), pull out strength of the self-drilling, self-tapping screws was similar to conventional self-tapping screws. The pull out forces required approximately 0.87 to 0.10 Newton kilograms, an insignificant difference. With cancellous bone blocks, the self-drilling screws had a three-fold increase in retentiveness when compared to the selftapping variety (Fig. 1). Thus the self-drilling, selftapping screws were essentially equal to the retentiveness of self-tapping screws in thin bone but were much superior in cancellous bone. This is presumably due to self-drilling screws compressing rather than cutting the cancellous bone around the threads of the screws.

Presented at the 1267th Meeting of the Keio Medical Society in Tokyo, July 15, 2002.

Reprint requests to: Dr. Brian Alpert, Oral \& Maxillofacial Surgery, Department of Surgical \& Hospital Dentistry, University of Louisville, School of Dentistry, Louisville, KY 40292, USA 

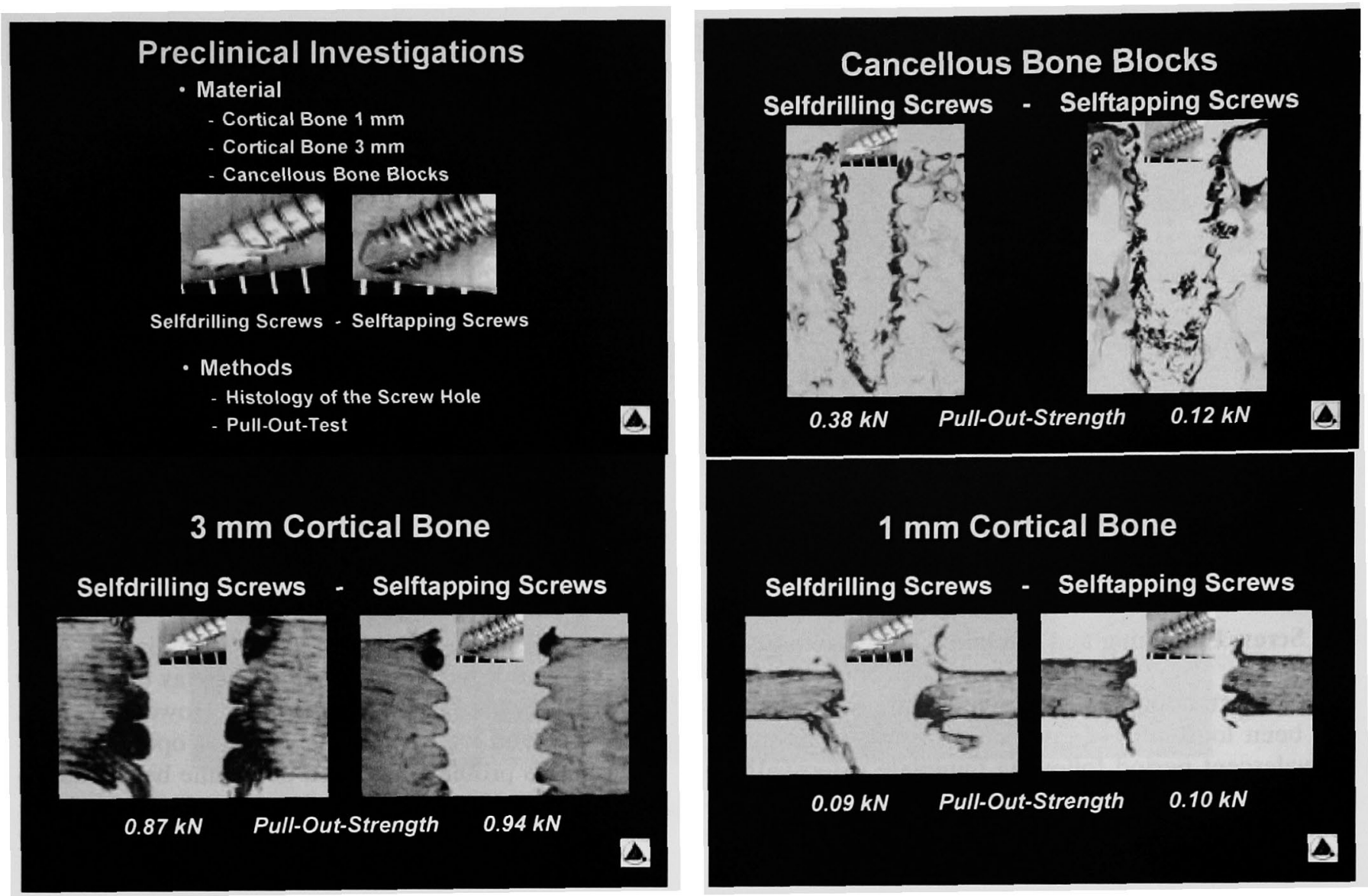

Fig. 1 Retention in cortical and cancellous bone.

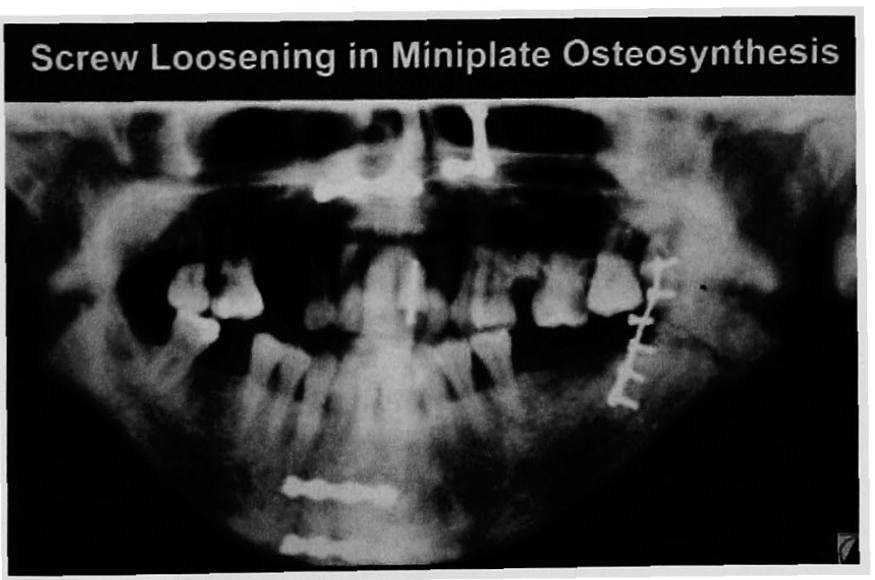

Fig. 2 Screw loosening.

The clinical implication is that self-drilling, self-tapping screws are much superior when fixing screws into a cancellous bone graft. The self-drilling principle also has the added advantage of avoiding the necessity to

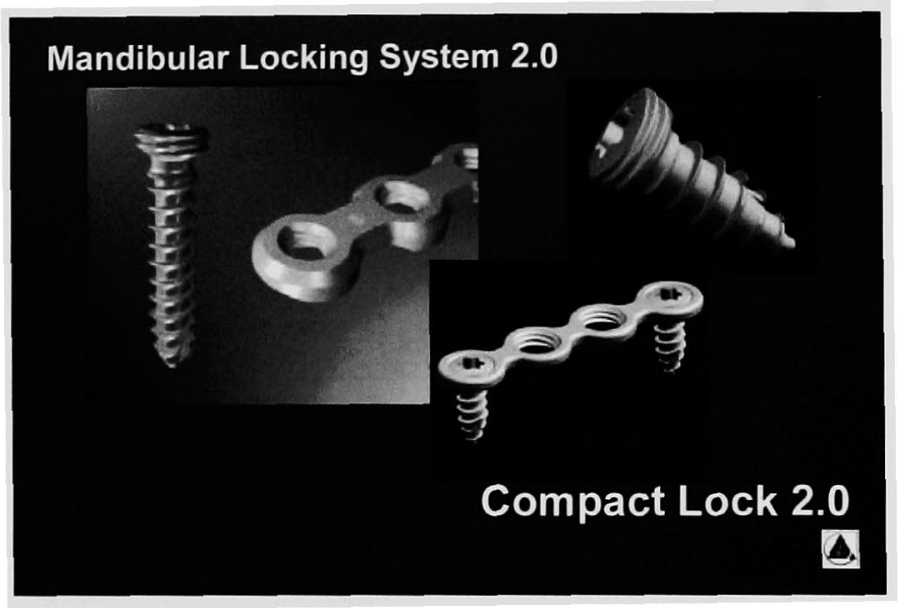

Fig. 3 Locking plate system.

drill a hole thus shortening the process of osteosynthesis and requiring less instrumentation. It should be noted that self-drilling screws may be difficult to apply in extremely dense bone such as the mandible. 


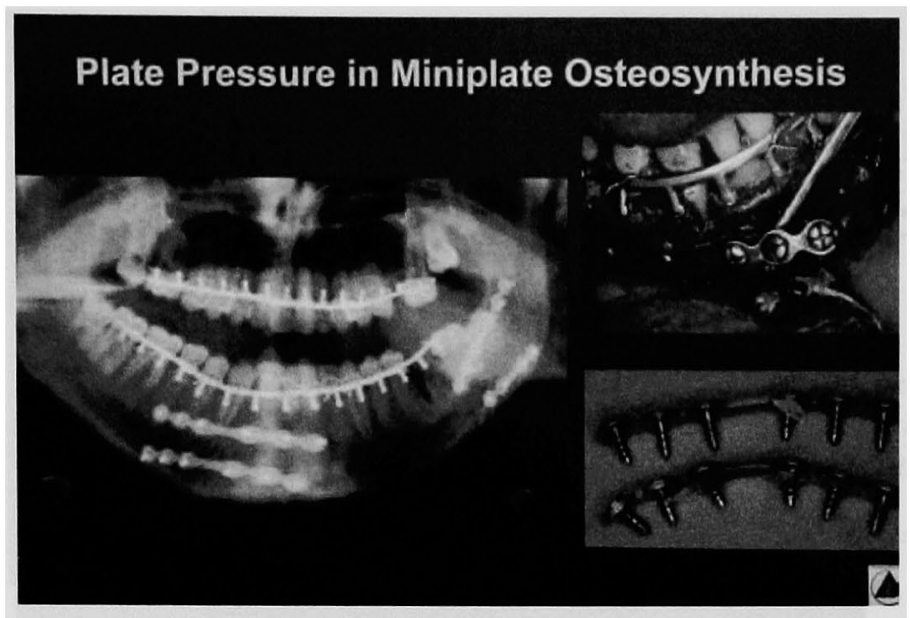

Fig. 4 Cortical necrosis under plate.

\section{Screw Loosening and Miniplate Osteosynthesis}

A long-standing problem in miniplate osteosynthesis has been loosening of one or more screws during the convalescent period following miniplate osteosynthesis (Fig. 2). This has been notably problematic in mandibular angle osteosynthesis where loosening screws

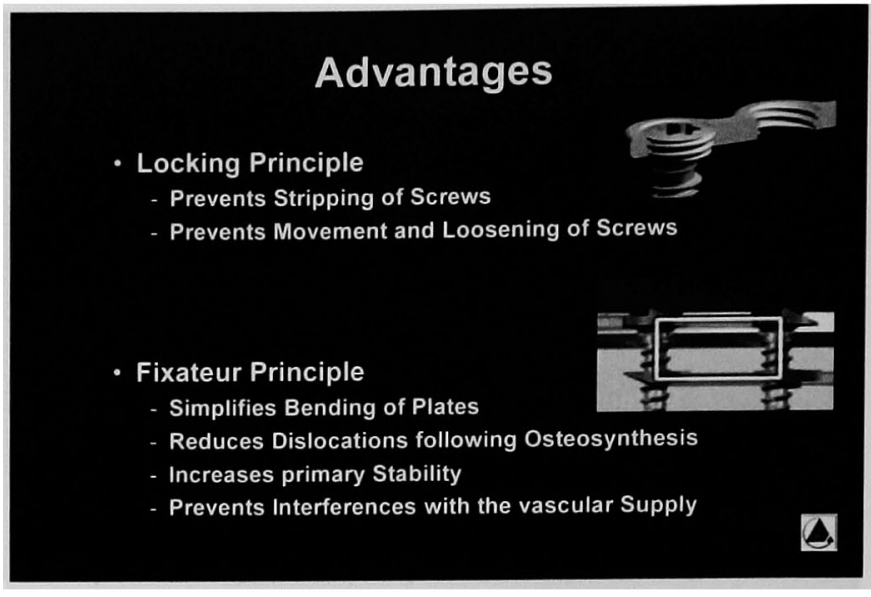

Fig. 5 Advantages of the locking system.

require removal of the rigid fixation appliance. As a rule this does not compromise the result as the fracture or osteotomy has healed underneath. ${ }^{1}$ However, it is an annoyance and leads to a second minor operative procedure. This problem has been overcome by the development of a screw which locks not only to the bone but to the bone plate (Fig. 3). This is accomplished by

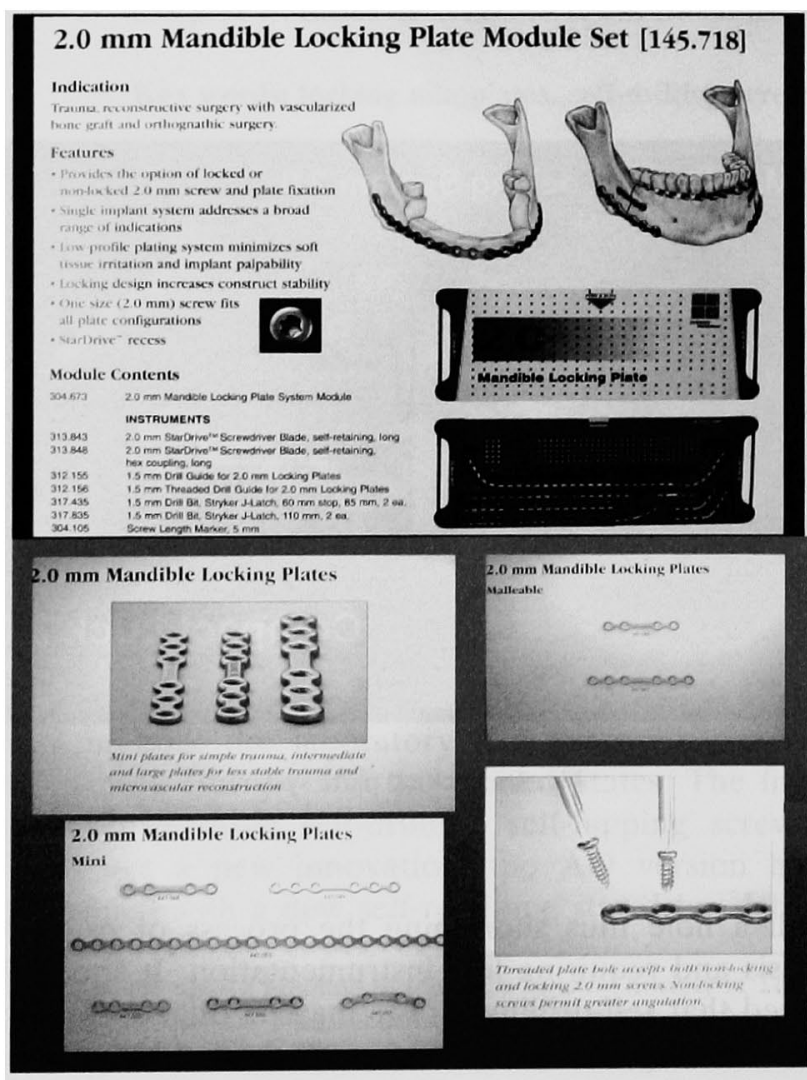

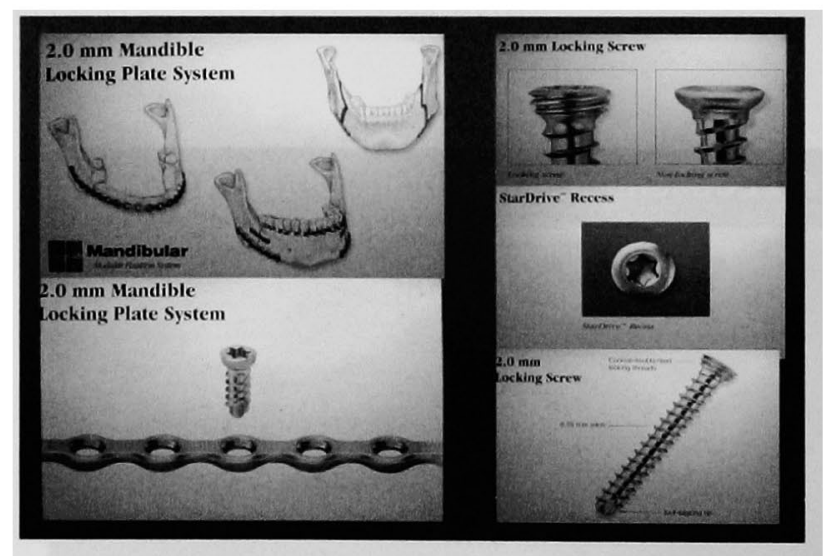

Fig. 6 The 2.0 locking mandible set. 


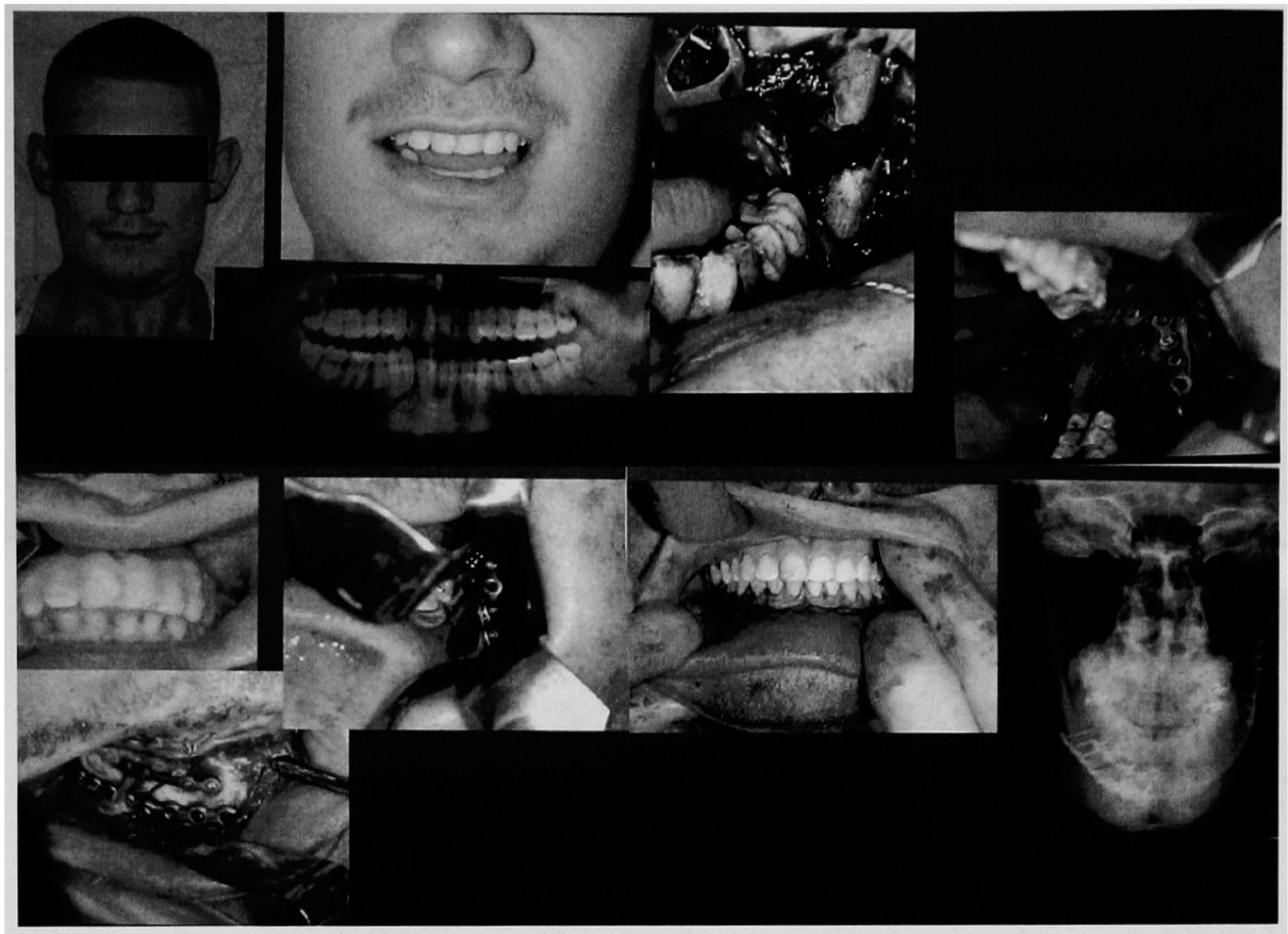

Fig. 7 Bilateral mandibular fracture managed with 2.0 locking miniplates and convalescent function.
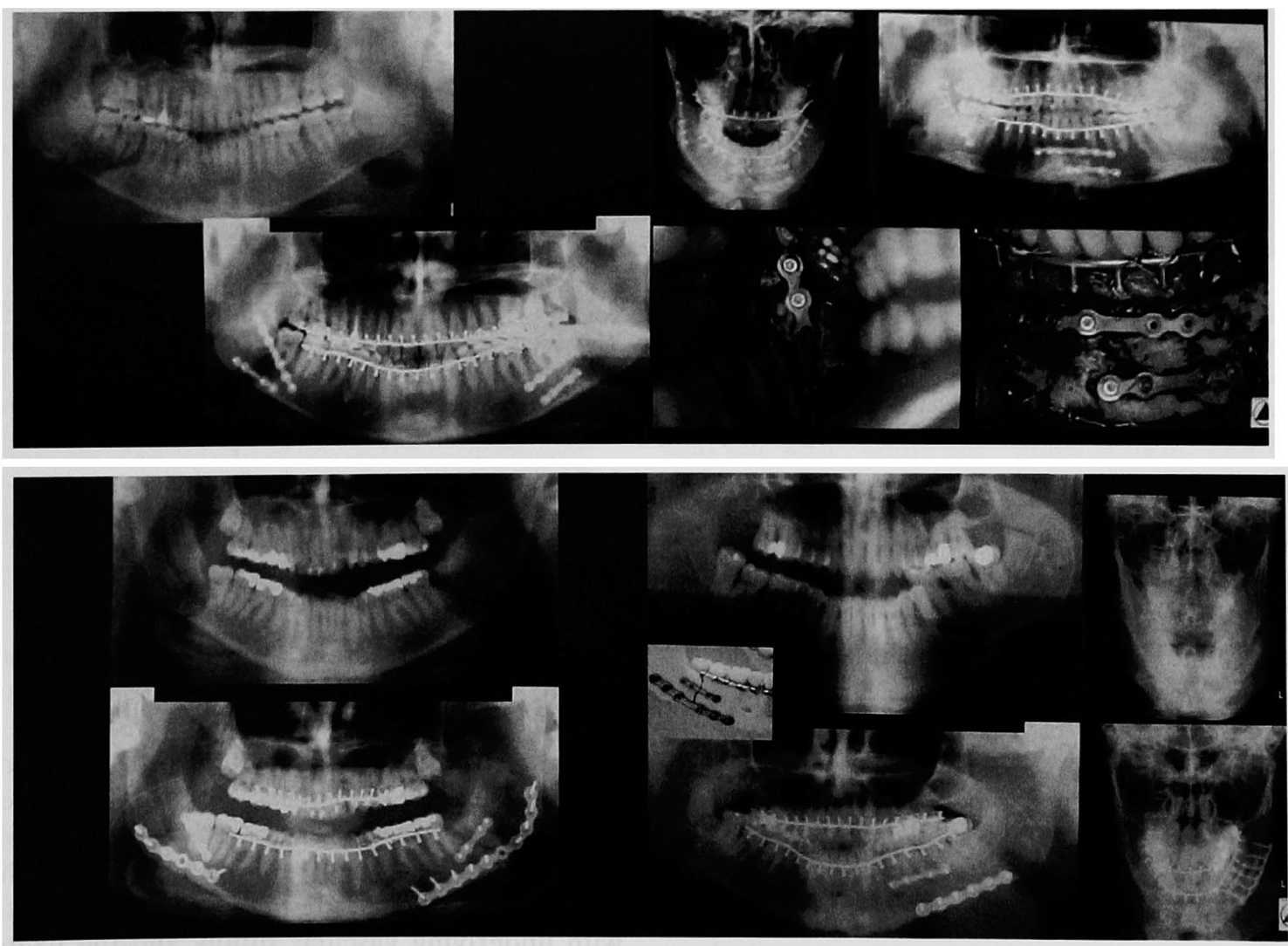

Fig. 8 Mandibular fractures managed with varying sizes and configurations of 2.0 locking miniplates. 

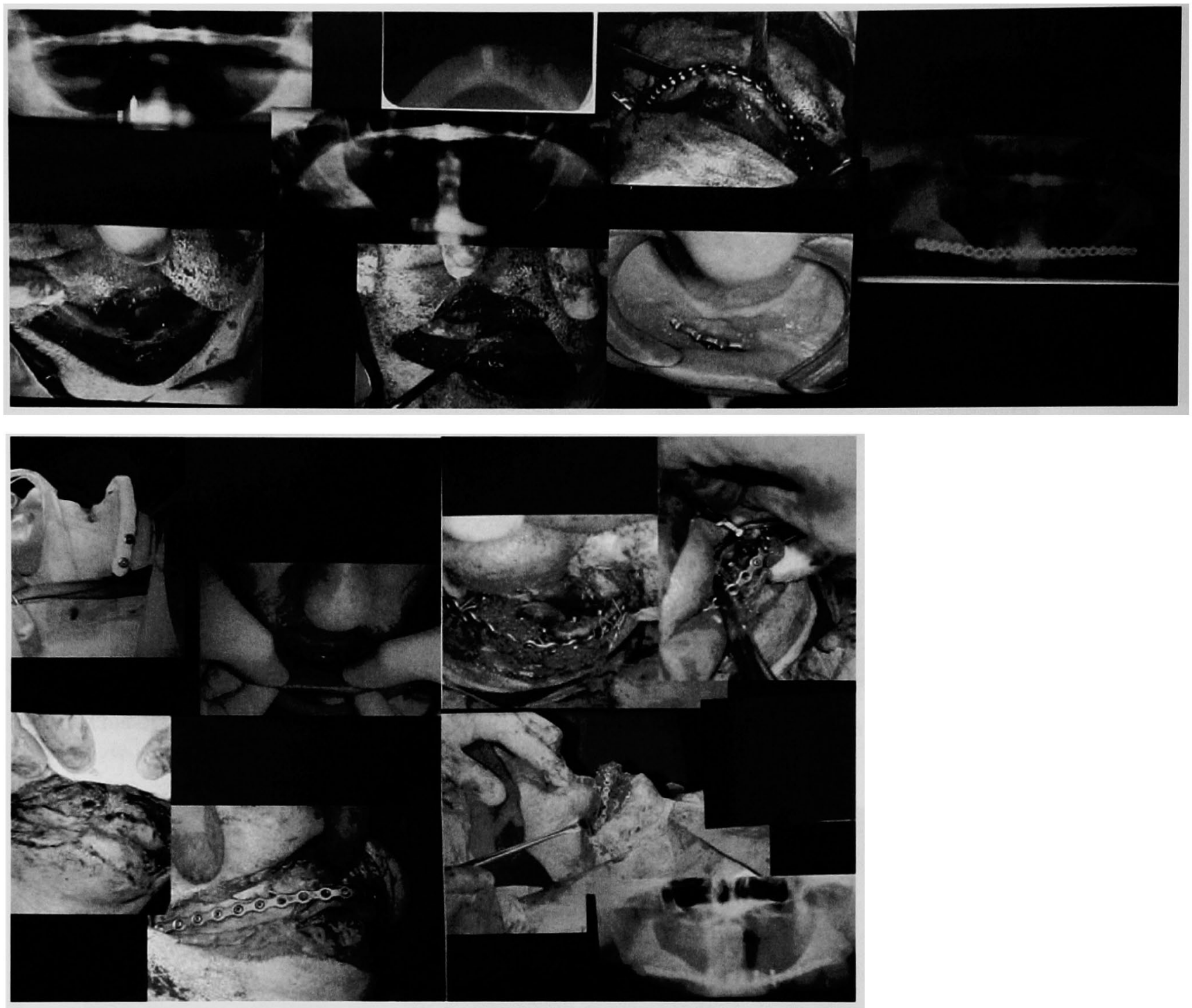

Fig. 9 Use of the 2.0 heavy locking reconstruction plate in support of reconstruction of a fibrous union of a mandibular symphysis fracture and in conjunction with bone grafts and primary dental implant placement. This patient sustained a pathologic fracture of his mandibular parasymphysis secondary to a failing dental implant. He was managed with a 2.4 locking reconstruction plate which later eroded into his mouth. The reconstruction plate was removed and skeletal pin fixation was placed. Further reconstruction some months later involved removing the external fixator, stabilizing the mandible with a 2.0 locking plate, placing primary tibial marrow bone grafts and dental implants.

having a screw with a double thread. One thread will engage the bone, another will engage a threaded area of the bone plate. The result is a locking plate system which in effect provides a mini-internal fixator.

Since the plate locks to the screw rather than gaining its rigidity by being compressed against the bone, it also avoids the cortical necrosis which is sometimes seen under a plate which is compressed against the bone (Fig. 4). Gutwald, et al., demonstrated the superiority of the locking plate utilizing cadaver studies. ${ }^{2}$

\section{Advantages of the Locking System}

The new locking system obviously had the advantage of not allowing the stripping of screws and prevented movement and loosening of screws. Since the plate did not have to be as precisely adapted to the underlying bone and indeed did not have to be compressed against the bone for stability, the bending of the plates was simplified. Dislocation following osteosynthesis was minimized or eliminated, there was less interference with underlying vascular supply and the fixator principle provided increased primary stability (Fig. 5). 

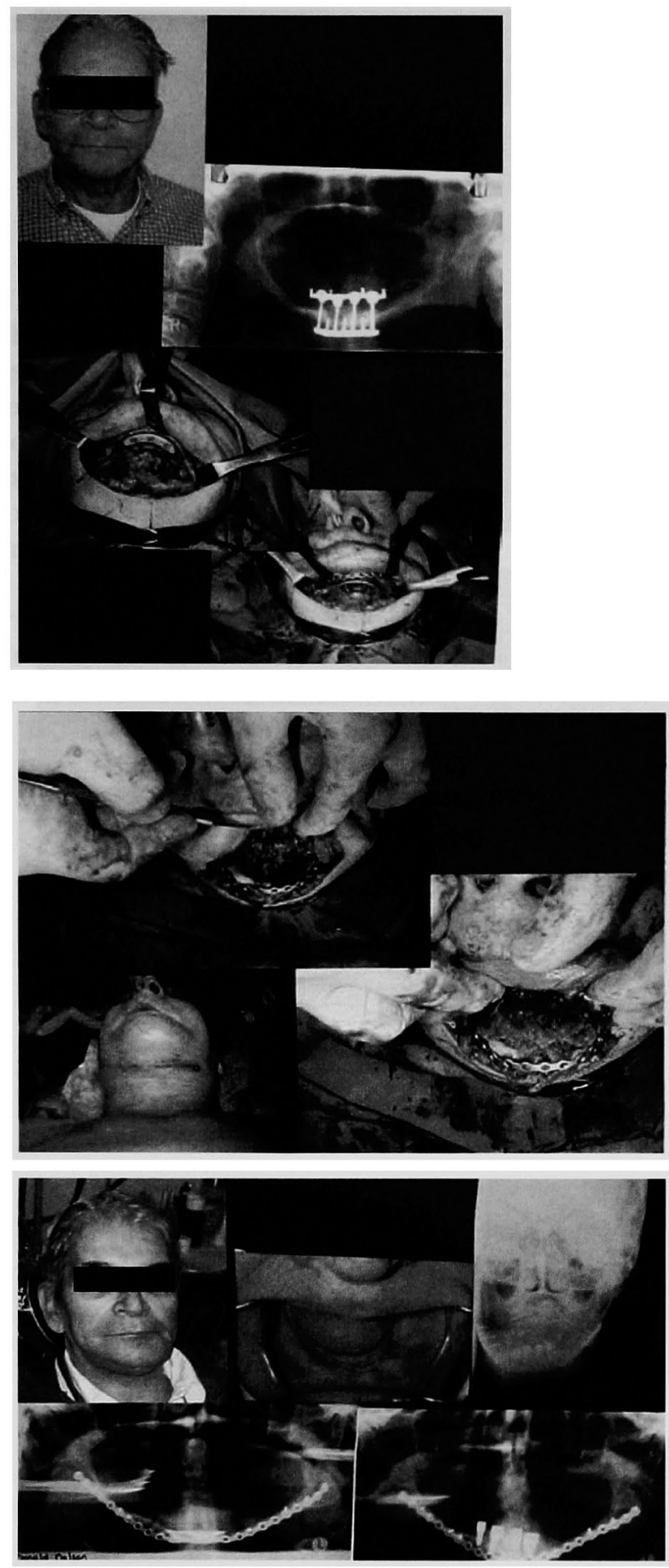

Fig. 1074 year old man with a failing staple implant. The patient heard a "crack" and $\mathrm{x}$-rays revealed a linear fracture through one of the postholes. At surgery the mandible was exposed from a submental approach, the mandible stabilized with a heavy 2.0 locking reconstruction plate, the failing stable implant removed, and the defects primarily grafted with autogenous marrow from the tibia. Three months later conventional dental implants were placed transorally.

\section{Clinical Trials}

Following preclinical studies, the system received extensive clinical trials in both European and North American centers. One center reported on their results. ${ }^{3}$ A variety of both manual and powered screw drivers were utilized in the clinical settings and several modifications were developed. It was noted that mandibular bone, particularly in older individuals could not be reliably drilled with a self-drilling screw. In such cases predrilling was necessary. It was also noted that a more malleable plate was needed for superior border plating of mandibular angle fractures. This was likewise developed and tested. The final product resulted from these extensive laboratory and clinical trials.

\section{Final Product}

Two systems were introduced as a result of these investigations. The 2.0 lock and 2.0 mandible system in Europe and the 2.0 mandible locking plate set in North America. The 2.0 lock system is useful in the management of midfacial fractures and osteotomies where a mini-internal fixator is desired. It is especially useful in the fixation of displaced zygomaticomaxillary complex fractures.

\section{The 2.0 Locking Mandible Set}

The 2.0 locking mandible set has received a great deal of attention in clinical trials (Fig. 6). The plates come in a variety of lengths and three separate thicknesses. Screws of varying lengths are provided as are appropriate instrumentation for bending, cutting and insertion. While the locking screws need to be centered and placed into the plate in perpendicular fashion, conventional screws can be utilized which may be placed at an angle. These obviously do not lock to the plate. The plates may be adapted with supplied bending pliers and cut with "short cut" plate cutters. The thin plates are available in either malleable or stiff forms allowing the operator to choose based on application.

\section{Use in Mandibular Fracture Repair}

The locking miniplates are especially useful in fixing fractures along lines of ideal osteosynthesis with miniplates and monocortical screws as popularized by Champy. They are not designed to replace the heavy bicortical systems, originally developed by Spiessl ${ }^{5}$ and Luhr. ${ }^{6}$ The monocortical screw-miniplate technique is well established. ${ }^{7}$ This is most useful in mandibular angle fractures in need of open reduction and internal fixation or in patients who require convalescent func- 

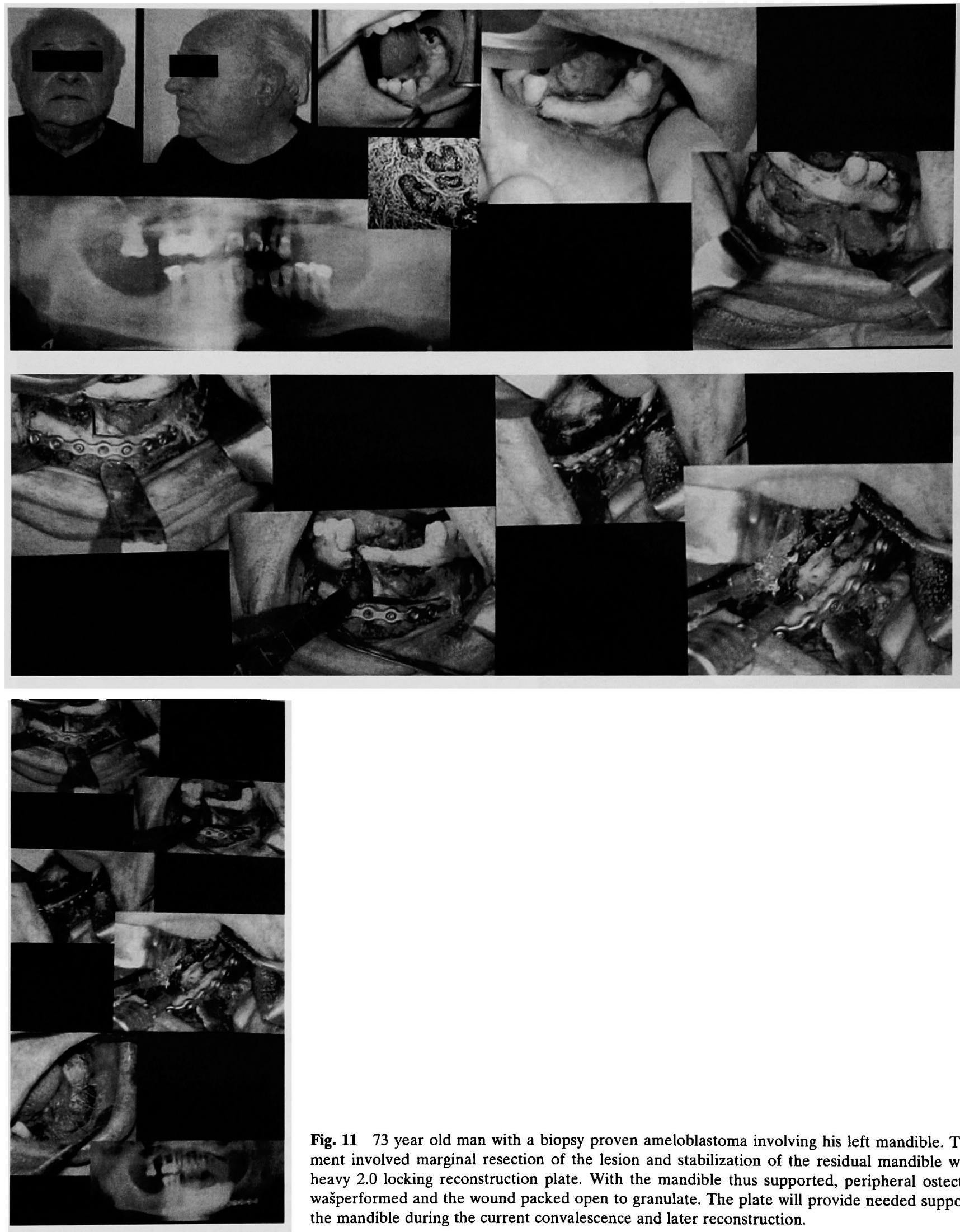

Fig. 1173 year old man with a biopsy proven ameloblastoma involving his left mandible. Treatment involved marginal resection of the lesion and stabilization of the residual mandible with a heavy 2.0 locking reconstruction plate. With the mandible thus supported, peripheral ostectomy wašperformed and the wound packed open to granulate. The plate will provide needed support to the mandible during the current convalescence and later reconstruction. 
tion. Examples are demonstrated in the accompanying illustrations (Fig. 7, 8). The medium and larger plates are useful where double plating is desired for fractures in the tooth bearing area.

\section{Aids to Reconstruction}

The larger, longer variety of locking miniplate can be a useful aid in selected areas of mandibular reconstruction. It is suitable for supporting the graft in free-flap reconstruction of the mandibular arch where opposing muscle forces are either absent or insignificant. It can also be useful in the management of unilateral atrophic mandibular fractures or in support of mandibles which are weakened by resection or atrophy both pending or during formal reconstruction with bone grafts. Three different cases illustrate possible uses (Fig. 9-11). It should be stressed that the 2.0 locking miniplate is not designed to replace the heavier 2.4 or 2.7 locking reconstruction plates designed for bilateral atrophic mandibular fractures or extensive reconstructions of the mandible.

\section{References}

1. Ellis E 3rd, Walker LR: Treatment of mandibular angle fractures using one noncompression miniplate. J Oral Maxillofac Surg 1996; 54: 864-872

2. Gutwald R, Alpert B, Schmelzeisen R: Principle and stability of locking plates. Keio J Med 2002; 51: 21-24

3. Ellis E 3rd, Graham J: Use of a 2.0-mm locking plate/screw system for mandibular fracture surgery. J Oral Maxillofac Surg 2002; 60 : 66442-66445

4. Champy M, Lodde JP, Schmitt R, Jaeger JH, Muster D: Mandibular osteosynthesis by miniature screwed plates via a buccal approach. J Maxillofac Surg 1978; 6: 14-21

5. Spiessl, B: New Concepts in Maxillofacial Bone Surgery. NY, Springer-Verlag, 1976

6. Luhr, HG: Compression plate osteosynthesis through the Luhr system. In: Kruger E, Schilli W, eds, Oral and Maxillofacial Traumatology, Vol. 1, Chicago, Quintessence Publishing Co, 1982

7. Alpert B, Engelstad M, Kushner GM: Invited review: small versus large plate fixation of mandibular fractures. J Craniomaxillofac Trauma 1999; 5: 33-39 